\title{
Beacon-based Opportunistic Scheduling in Wireless Body Area Network
}

\author{
Yang Zhou ${ }^{1}$, Student Member, IEEE, Zhengguo Sheng ${ }^{2}$, Member, IEEE, \\ Victor C. M. Leung ${ }^{1}$, Fellow, IEEE, and Peyman Servati ${ }^{1}$, Member, IEEE
}

\begin{abstract}
Wireless Body Area Networks (WBANs) are one of the key technologies that support the development of digital health care, which has attracted increasing attention in recent years. Compared with general Wireless Sensor Networks (WSNs), WBANs have more stringent requirements on reliability and energy efficiency. Though WBANs are applied within limited transmission range, the on-body channel condition can be very challenging because of blocking or absorbing of signal. In this paper, we are looking into the design of Medium Access Control (MAC) protocols and propose an opportunistic scheduling scheme by applying heuristic scheduling and dynamic superframe length adjustment to improve the system performance. The simulations have been supplemented to show the advantages of the proposed solutions in outage rate performance, compared with existing solutions.
\end{abstract}

\section{INTRODUCTION}

Inspired by the development of Internet-of-Things [5], [6], Wireless Body Area Networks (WBANs), which collect and transfer real time body signals for medical care purpose, play a fundamental role in future health applications. In such a network, energy efficiency and transmission reliability are major concerns. Reducing outage rate is thus crucial since it indicates less retransmissions, which will save energy and reduce transmission delay.

Though WBANs are applied within limited range, the unpredictable channel environment through human body is a challenge for wireless communication [2]. The reason is that the transmission power of sensors need to keep low to save energy and avoid impairing body tissues. At the same time, body parts can block and absorb the signals, making the link status vulnerable. Opportunistic scheduling can take channel state into consideration and since the channel fluctuation is a major influence on the performance of WBANs, it has the potential of improving the system performance to a large extent by selecting good channels.

A survey [1] provides a comprehensive summary on opportunistic scheduling. So far, the opportunistic scheduling research largely focuses on cellular networks, which are not necessarily suitable for WBANs. WBANs have special requirements of extremely low energy consumption and high reliability that are not considered by the methods in [1].

Several solutions have been proposed focusing on opportunistic scheduling to WBANs. [8], [9], [11] focus on applying Markov chain model to forecast the channel condition based on the previous transmissions. In [8], [9], the

\footnotetext{
${ }^{1}$ Department of Electrical and Computer Engineering, the University of British Columbia, Vancouver, BC Canada \{yangzhou, vleung, peymans\}eece.ubc.ca

${ }^{2}$ Department of Engineering and Design, the University of Sussex, U.K. z.shengesussex.ac.uk
}

two-state Gilbert model is used to analyze the link status of WBANs. A flipping strategy is proposed and proven to perform better than the other alternatives. However its assumption of homogeneous link status among all sensor nodes is not realistic. The flipping strategy can be misleading when the speed of different channels converging to the steady states varies greatly. In [11], similarly, the authors propose a threshold-based scheduling scheme, which is also based on Gilbert model and monotonicity property. However, no method of acquiring the transition matrix is provided, hence the schedule made accordingly cannot be reliable.

In this paper, we propose a novel transition matrix estimation method by taking channel dynamics into consideration. Furthermore, we design a heuristic scheduling method that is proven to be optimal under some minor assumptions. Based on our observation that the superframe length plays a significant role on the performance of opportunistic scheduling, we further propose to dynamically adjust the superframe length according to the channel status. As shown by the simulation, the proposed method can reduce $9 \%$ of the outage occurrences compared with the fixed scheduling, which turns out to be much better than the existing random method and the flipping method [8], [9].

The rest of the paper is organized as follows. In Section II, we introduce the system model and formulate the scheduling problem in WBAN context. In Section III and IV, we present our heuristic scheduling scheme and dynamic superframe length adjustment approach. The simulation results are presented in Section V and Section VI concludes the paper.

\section{SYSTEM MODEL AND PROBLEM FORMULATION}

\section{A. System Model}

We focus on scenarios such as health care and fitness. Sensor nodes locate on the surface of human body and transmit the data of life signals to the coordinator periodically. A single-hop star topology is adopted. Sensor nodes are generally powered by tiny batteries and of limited capacity. The coordinator is an ordinary node, such as cell phone. Both IEEE 802.15.4 and IEEE 802.15.6, which are widely used for WBANs suggest a beacon-enabled TDMA working mode. A beacon frame is broadcasted by coordinator for synchronization and resource allocation at the beginning of each superframe. Each node will wake up and transmit in assigned time slots in each superframe periodically.

The Gilbert model [4] is adopted, where there are two channel states defined for each time slot, namely "good" and "bad". They are represented by " 1 " and "0" respectively. 
Because energy is extremely limited in WBANs, probing the channels is not suitable. The only chance to observe the channel status for the coordinator is when a node is transmitting. At the other time slots, we use state belief instead of the exact channel status to measure the channel condition. If the transition matrix for node $i$ is $\mathbf{P}^{i}=\left[\begin{array}{ll}p_{00}^{i} & p_{01}^{i} \\ p_{10}^{i} & p_{11}^{i}\end{array}\right]$ and the initial state belief of node $i$ to be $\left[\begin{array}{l}p_{0}^{i}(0) \\ p_{1}^{i}(0)\end{array}\right]$, the state belief followed at time slot $n$ can be calculated as

$$
\left[\begin{array}{l}
p_{0}^{i}(n) \\
p_{1}^{i}(n)
\end{array}\right]^{T}=\left[\begin{array}{l}
p_{0}^{i}(0) \\
p_{1}^{i}(0)
\end{array}\right]^{T}\left[\begin{array}{cc}
p_{00}^{i} & p_{01}^{i} \\
p_{10}^{i} & p_{11}^{i}
\end{array}\right]^{n}
$$

where $p_{0}^{i}(0)$ is the probability of having a bad channel at "slot 0 " and $p_{1}^{i}(0)$ is that of a good channel. "slot 0 " is when transmission happens and the channel condition is known.

\section{B. Problem Formulation}

The objective is to maximize the average number of successfully transmitted packets in each superframe. Assume $h_{i}(\tau)$ represents the channel quality, which is the packet delivery rate of node $i$ at time slot $\tau$ in a superframe and we can express the problem as

$$
\begin{array}{cc}
\text { maximize } & \sum_{1 \leq i \leq M, 1 \leq \tau \leq T} h_{i}(\tau) I_{i}(\tau) \\
\text { subject to } & \sum_{1 \leq i \leq M} I_{i}(\tau) \leq 1 \\
& \sum_{1 \leq \tau \leq T} I_{i}(\tau)=x_{i}
\end{array}
$$

where $I_{i}(\tau)$ is an indicator function representing whether node $i$ is scheduled at slot $\tau$ and $x_{i}$ represents the number of packets node $i$ needs to transmit in each superframe. $M$ represents the number of nodes. $T$ is the number of slots in each superframe. Here we assume only one packet is transmitted in a slot.

If we have reliable estimations of $h_{i}(\tau)$, this problem can be turned into maximum weighted bipartite matching problem. Hungarian algorithm gives a solution of complexity $O\left(T^{3}\right)$. However, the value can be quite large for the computation per superframe since $T$ can be hundreds of slots.

\section{HEURISTIC SCHEDULING}

\section{A. Transition Matrix Estimation}

The existing Markov models that focus on WBANs assume stationary channels, that is, the transition probabilities are constants, such as [8], [9], [11] and many works mentioned in [1]. However, considering the highly dynamic channel environment of WBANs, we take the fluctuation of transition matrix into consideration and estimate the probabilities by state transition frequencies. We only assume the transition matrix stays the same during the time range of scheduling at the beginning of each superframe.

The coordinator observes and calculates the frequency of each node transitioning from one state to another to approximate the transition probabilities. For node $i$, the coordinator will calculate $W_{S_{1} S_{2}}^{i}(r)$, which is the weighted sum of the times that node $i$ transits from state $S_{1}$ to state $S_{2}$ until the $r^{t h}$ superframe, $S_{1}, S_{2} \in\{0,1\}$. Specifically, we define a discounting factor $\rho$ and a time period length $L$. $W_{S_{1} S_{2}}^{i}(r)$ will be discounted by a factor of $\rho$ every $L$ superframes. The weighted sum is used so that we give more trust to the recent samples and less to the remote ones. Then for the $r^{t h}$ superframe, we have the estimation $p_{S_{1} S_{2}}^{i}(r)=\frac{W_{S_{1} S_{2}}^{i}(r)}{\sum_{S} W_{S_{1} S}^{i}(r)}$, where $S \in\{0,1\}$.

\section{B. Heuristic Scheduling}

One basic idea is to divide the nodes into two groups. One group contains the nodes that succeed their last transmissions in the previous superframe and the other contains the failed ones. The "successful" nodes should be scheduled ahead of the "failed" nodes. In this way, the node with a good channel is able to take advantage of the channel before it turns to worse, and the node with a bad channel will have enough time to recover [8].

According to Section II, we can calculate $p_{1}^{i}$ to approximate $h_{i}$ in the objective function, where the last transmission in the previous superframe is the initial state belief $p_{1}^{i}(0)$. According to [7], the coherence time of BAN channel is around $400 \mathrm{~ms}$, which is generally larger than the superframe length in our design. So the channel belief we estimate is reliable. It can be proved that $p_{1}^{i}(t)$ evolves as an exponential function as (3) [8]. To simplify the expression and according to the fact that the channel dynamics during scheduling can be regarded as stationary, we omit the superframe index $r$ and use $\varepsilon_{i}$ to represent the probability of transitioning from bad channel to good channel $p_{01}^{i}(r)$ and $\delta_{i}$ to represent the probability of transitioning from good channel to bad channel $p_{10}^{i}(r)$. The belief of having a good channel at time $t$ can be expressed as (3). $t$ is the number of time slots away from the initial state, which is the result of the previous transmission.

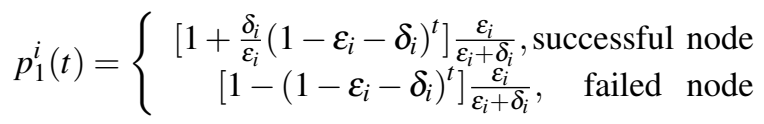

We use the fluctuation range of channel belief to be the utility function or the heuristic function. Specifically, for successful nodes, we calculate the channel belief difference of each node between the start of one superframe and the end of all successful nodes' transmissions. Assume $N_{i}$ is the number of time slots between the previous transmission of node $i$ and the beginning of current superframe and $N_{\text {Good }}$ is the number of time slots assigned for successful nodes' transmissions. The utility function for the successful nodes can be expressed as

$$
U_{i}=p_{1}^{i}\left(N_{i}\right)-p_{1}^{i}\left(N_{i}+N_{\text {Good }}\right)
$$

The node with a larger utility function will be scheduled in the front. This design can be illustrated as in Figure 1. Assume there are 4 nodes and node 1 fails the transmission while the others succeed.

Similarly, the utility function for the failed nodes is

$$
U_{i}=p_{1}^{i}\left(N_{i}+T-N_{\text {Bad }}\right)-p_{1}^{i}\left(N_{i}+T\right)
$$






Fig. 1. Illustration on heuristic scheduling

where $N_{B a d}$ is the number of time slots assigned to the failed nodes and $T$ is the number of time slots in one superframe.

Lemma 1 Given that node $i$ and node $j$ are in the same group (both fail or succeed in their previous transmissions), if $\varepsilon+\delta$ are identical among all nodes and $U_{i}>U_{j}$, the slots of node $i$ should be scheduled in front of the slots of node $j$.

See Appendix for detailed proof of Lemma 1. Assume each node transmits $N_{p}$ packets in each superframe on average, the complexity of the proposed scheduling scheme is $O\left(M \times N_{p}+M \log M\right)$. This computational complexity is trivial for a powerful coordinator in WBANs, particularly for normal cases with a limited number of sensor nodes and low working load. The flipping method in [8] is a special case of our proposed method, where both $\varepsilon$ and $\delta$ are required to be identical for all nodes, as illustrated in Appendix.

\section{DYNAMIC SUPERFRAME LENGTH ADJUSTMENT}

We propose a dynamic superframe length adjustment method that can adjust the superframe length online according to the channel condition. The problem of deciding the superframe length can be formulated as a decision problem, where the agent is the coordinator and it makes decisions based on the states and rewards. The states, actions and rewards can be defined as

1) State: Packet Delivery Rate (PDR) in the previous superframe

2) Action: Choice of the superframe length $T$ and the number of packets to deliver in one superframe $x_{i}$

3) Reward: $r w=-(1-\mathrm{PDR})+\mathrm{PDR}$ in the current superframe

We use action-value function $Q(s, a)$, which represents the expected value of performing action $a$ in state $s$, to make decisions. In each state, we will pick the action with the largest $Q$ value being $\varepsilon$-greedy. That is, we choose the action with the largest $Q$ value with probability $1-\varepsilon$ and choose a random action for exploration with probability $\varepsilon$. According to Q-learning, the updating rule of $Q$ values is [10]

$$
Q(s, a) \leftarrow Q(s, a)+\alpha\left[r w+\gamma \max _{a^{\prime}} Q\left(s^{\prime}, a^{\prime}\right)-Q(s, a)\right]
$$

The pseudo code of this method is in Algorithm 1.

\section{SIMULATION RESULTS}

In the simulation, there are five sensor nodes and one coordinator, which are on the surface of human body. Each time slot is $5 \mathrm{~ms}$ and the number of packets transmitted



by each node in each superframe is identical. The random scheduling and flipping strategy [8], [9] are used as comparisons. The percentage of the outage occurrences avoided compared with fixed scheduling is used as the evaluation criteria. The channel gain samples of on-body channels from data set [3] are used and the results are averaged over 8 data sets of different persons. The default value $T_{0}$ of the proposed method, which equals to the fixed superframe length of the other methods, is set to be 40 slots. There are five superframe length values defined for dynamic superframe length scheduling, which are 40, 80, 120, 160 or 200 slots.

In Figure 2, the influence of packet number is shown. The number of packets per superframe for the fixed superframe length methods ranges from 2 to 7 and it is the same for the default packet number $x_{i 0}$ of the proposed method. The outage threshold is $-80 \mathrm{~dB}$. It is obvious that a combination of the proposed heuristic scheduling and variable superframe length scheme yields the best performance. As the number of packets per superframe increases, the performance of all methods deteriorates. The reason is when the transmissions become more crowded within one superframe, it is harder to schedule and avoid the bad channel status. In Figure 3, the influence of outage threshold is presented. The combined heuristic scheduling and variable superframe length scheme can achieve $8 \%-9 \%$ less outages compared with the fixed scheduling. As comparisons, the random method can only achieve $5 \%-6 \%$ and flipping $6 \%-7 \%$.

It is worth noting that no extra overhead is added to the 


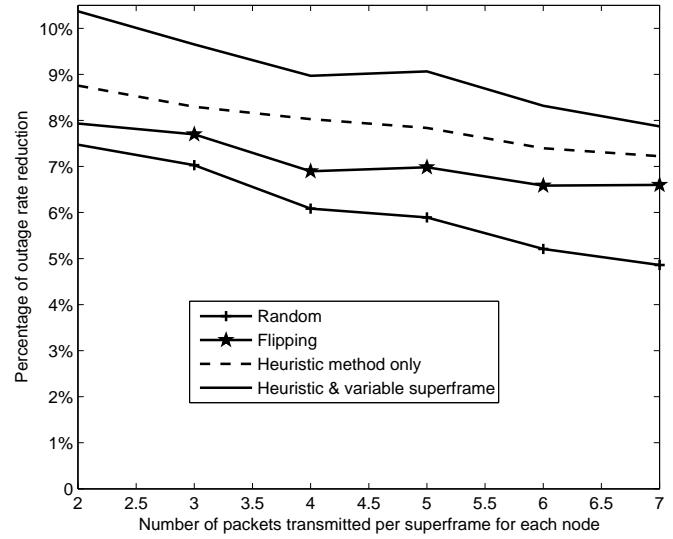

Fig. 2. Influence of packet number



Fig. 3. Influence of outage threshold

sensor nodes in our proposed method. All calculation is done by the coordinator, which is not power constrained.

\section{CONCLUSION}

There are two major contributions in this paper. Firstly, we have proposed a method to estimate the channel dynamics and further, based on this estimation, we have designed a simple scheduling scheme with good performance using the proposed heuristic function. Secondly, we have revealed the fundamental effect of a proper superframe length in opportunistic scheduling. Our combined scheduling yields a much better performance on the outage rate compared with the literature.

\section{APPENDIX}

Consider two nodes $i$ and $j$ that are both in the "successful" group (the failed nodes can be proved with the same process). If node $i$ has a larger utility function than node $j$, from (3) and (4) we can get

$$
\begin{aligned}
& \left(1-\varepsilon_{i}-\delta_{i}\right)^{N_{i}} \times \frac{\delta_{i}}{\varepsilon_{i}+\delta_{i}} \times\left[1-\left(1-\varepsilon_{i}-\delta_{i}\right)^{N_{G o o d}}\right] \\
& >\left(1-\varepsilon_{j}-\delta_{j}\right)^{N_{j}} \times \frac{\delta_{j}}{\varepsilon_{j}+\delta_{j}} \times\left[1-\left(1-\varepsilon_{j}-\delta_{j}\right)^{N_{G o o d}}\right]
\end{aligned}
$$

If $\varepsilon_{i}+\delta_{i}=\varepsilon_{j}+\delta_{j}$, we have

$$
\left(1-\varepsilon_{i}-\delta_{i}\right)^{N_{i}} \times \frac{\delta_{i}}{\varepsilon_{i}+\delta_{i}}>\left(1-\varepsilon_{j}-\delta_{j}\right)^{N_{j}} \times \frac{\delta_{j}}{\varepsilon_{j}+\delta_{j}}
$$

Assume node $i$ and node $j$ are scheduled at time slot $t_{i}$ and $t_{j}$ in the current superframe respectively. If $t_{i}>t_{j}$, that is, node $i$ is scheduled behind node $j$, then from

$$
\begin{aligned}
& {\left[p_{1}^{i}\left(N_{i}+t_{j}\right)+p_{1}^{j}\left(N_{j}+t_{i}\right)\right]-\left[p_{1}^{i}\left(N_{i}+t_{i}\right)+p_{1}^{j}\left(N_{j}+t_{j}\right)\right]} \\
& =\left[\frac{\delta_{i}}{\varepsilon_{i}+\delta_{i}} \times\left(1-\varepsilon_{i}-\delta_{i}\right)^{N_{i}}-\frac{\delta_{j}}{\varepsilon_{j}+\delta_{j}} \times\left(1-\varepsilon_{j}-\delta_{j}\right)^{N_{j}}\right] \\
& \times\left[\left(1-\varepsilon_{i}-\delta_{i}\right)^{t_{j}}-\left(1-\varepsilon_{i}-\delta_{i}\right)^{t_{i}}\right]>0
\end{aligned}
$$

we can conclude swapping the slots assigned to node $i$ and node $j$ can improve the expectation of the amount of successfully transmitted packets when node $i$ is scheduled behind node $j$ and $U_{i}>U_{j}$. So node $i$ should be scheduled in front of node $j$ instead. It is worth noting that if $\varepsilon_{i}=\varepsilon_{j}$ and $\delta_{i}=\delta_{j}$, (8) becomes $N_{i}<N_{j}$ and the proposed method is equivalent to the flipping method [8].

\section{ACKNOWLEDGMENT}

This work was supported by the Canadian Natural Sciences and Engineering Research Council.

\section{REFERENCES}

[1] Arash Asadi and Vincenzo Mancuso. A survey on opportunistic scheduling in wireless communications. Communications Surveys \& Tutorials, IEEE, 15(4):1671-1688, 2013.

[2] Athanassios Boulis, David Smith, Dino Miniutti, Lavy Libman, and Yuriy Tselishchev. Challenges in body area networks for healthcare: The mac. Communications Magazine, IEEE, 50(5):100-106, 2012.

[3] D. Rodda B. Gilbert J. Dong D. Smith, L. Hanlen and V. Chaganti. Body area network radio channel measurement set. http://filestore.nicta.com.au/Comms/OpenNICTA/ NICTA_BodyAreaNetwork_RadioChannel_Data/. Accessed 10-Dec-2015.

[4] Edgar N Gilbert. Capacity of a burst-noise channel. Bell system technical journal, 39(5):1253-1265, 1960.

[5] Zhengguo Sheng, Chinmaya Mahapatra, Chunsheng Zhu, and Victor Leung. Recent advances in industrial wireless sensor networks toward efficient management in iot. Access, IEEE, 3:622-637, 2015.

[6] Zhengguo Sheng, Hao Wang, Changchuan Yin, Xiping Hu, Shusen Yang, and Victor Leung. Lightweight management of resourceconstrained sensor devices in internet of things. Internet of Things Journal, IEEE, 2(5):402-411, 2015.

[7] David B Smith, Athanassios Boulis, and Yuriy Tselishchev. Efficient conditional-probability link modeling capturing temporal variations in body area networks. In Proceedings of the 15th ACM international conference on Modeling, analysis and simulation of wireless and mobile systems, pages 271-276. ACM, 2012.

[8] Yuriy Tselishchev, Athanassios Boulis, and Lavy Libman. Variable scheduling to mitigate channel losses in energy-efficient body area networks. Sensors, 12(11):14692-14710, 2012.

[9] Yuriy Tselishchev, Lavy Libman, and Athanassios Boulis. Reducing transmission losses in body area networks using variable tdma scheduling. In World of Wireless, Mobile and Multimedia Networks (WoWMoM), 2011 IEEE International Symposium on a, pages 1-10. IEEE, 2011.

[10] Christopher John Cornish Hellaby Watkins. Learning from delayed rewards. PhD thesis, University of Cambridge England, 1989.

[11] Zhisheng Yan, Bin Liu, and Chang Wen Chen. Qos-driven scheduling approach using optimal slot allocation for wireless body area networks. In e-Health Networking, Applications and Services (Healthcom), 2012 IEEE 14th International Conference on, pages 267-272. IEEE, 2012. 